\title{
Interaction of cavity solitons in degenerate optical parametric oscillators.
}

\author{
Dmitry V. Skryabin and William J. Firth \\ Department of Physics and Applied Physics, University of Strathclyde, Glasgow, G4 ONG, Scotland
}

(June 29, 2006)

\begin{abstract}
Numerical studies together with asymptotic and spectral analysis establish regimes where soliton pairs in degenerate optical parametric oscillators fuse, repel, or form bound states. A novel bound state stabilized by coupled internal oscillations is predicted.
\end{abstract}

Many nonlinear media can support soliton-like structures when contained in a driven optical cavity $[1,4$. We will refer to such structures as cavity solitons (CS). In quadratic nonlinear media CS have recently been predicted in both optical parametric oscillator (OPO) [3] and second harmonic generation [4] configurations. Although experimental observation of $\chi^{(2)}$-CS remains a challenge, impressive bistability results [5] demonstrate the required level of nonlinearity and thus pave the way towards this goal.

The large values of effective $\chi^{(2)}$ accessible in artificially phase-matched materials in combination with their practically instantaneous response are important advantages of using quadratic nonlinearity for implementation of CS for all-optical processing of information. They thus represent an interesting alternative to the CS which can be created in cavities with dispersive-absorptive [i] , and resonant electron-hole [2] types of nonlinearities. In all such schemes high CS density is desirable and therefore understanding of their interaction is a practically important problem which is still largely open. In this Letter we focus on the interactions of CS found in the belowthreshold regime of a degenerate doubly resonant OPO, under conditions where the signal field has three coexistent plane-wave states [3].

Assuming phase-matching, a plane-wave input field, and ignoring walk-off, the mean-field OPO equations can be presented in the following dimensionless form [3]

$$
\begin{aligned}
& -i \partial_{t} E_{1}=\left(\alpha_{1} \partial_{x}^{2}+\delta_{1}+i \gamma_{1}\right) E_{1}+\left(E_{2}+\mu\right) E_{1}^{*}, \\
& -i \partial_{t} E_{2}=\left(\alpha_{2} \partial_{x}^{2}+\delta_{2}+i \gamma_{2}\right) E_{2}+E_{1}^{2} / 2
\end{aligned}
$$

Here $E_{1}$ and $\left(E_{2}+\mu\right)$ are the signal and pump fields, respectively, at frequencies $\omega$ and $2 \omega$ (we use $\mu$ as a measure of the pump strength). The slow time $t$ is scaled so that $\gamma_{m}$ (proportional to the cavity damping rates) and $\delta_{m}$ (to the detunings from its resonances) are of order unity. Here and below $m=1,2$.

This system can describe either diffractive or dispersive effects. We consider $x$ a dimensionless transverse coordinate, and so set $\alpha_{m}=1 / m$. For this case, existence of CS for $\delta_{m}<0$ was numerically demonstrated [3] for $\mu_{L}<\mu<\mu_{R}$, where $\mu_{L}=\left|\gamma_{1} \delta_{2}+\gamma_{2} \delta_{1}\right| / \sqrt{\delta_{2}^{2}+\gamma_{2}^{2}}$, and $\mu_{R}=\sqrt{\delta_{1}^{2}+\gamma_{1}^{2}}$ is the OPO threshold. Within this range two different non-trivial homogeneous solutions $\left(E_{m} \neq 0\right.$, $\left.\partial_{x} E_{m}=0\right)$ coexist with the trivial one $\left(E_{m}=0\right)$, and the CS are sech-like localized states on the zero background.

We start our analysis by applying a perturbative method [6] to the problem of CS interaction. We seek solutions of Eqs. (1) in the form

$$
\begin{aligned}
& E_{m}(x, t)=A_{m}\left(x-x_{A}\right)+B_{m}\left(x-x_{B}\right)+ \\
& \epsilon\left(a_{m}\left(x-x_{A}, x_{B}, t\right)+b_{m}\left(x-x_{B}, x_{A}, t\right)\right)+O\left(\epsilon^{2}\right),
\end{aligned}
$$

where $A_{m}\left(x-x_{A}\right)$ and $B_{m}\left(x-x_{B}\right)$ are CS centred on $x_{A, B}$. Note that Eqs. (11) are invariant with respect to a $\pi$ phase flip of the signal field, so that $A$ and $B$ can be either in-phase or out-of-phase CS. We assume $0<\epsilon \ll 1$, and that the perturbation functions $a_{m}, b_{m}$ are negligible except close to $x_{A}, x_{B}$ respectively. We further assume that $x_{A, B}$ vary on the slow time scale $\tau=\epsilon t$ and that $d=\left|x_{A}-x_{B}\right|$ is large enough that the overlap functions $\mathcal{I}_{1}=A_{2} B_{1}^{*}+B_{2} A_{1}^{*}$ and $\mathcal{I}_{2}=A_{1} B_{1}$ are of order $\epsilon$.

Substituting ansatz (2) into Eqs. (11) and truncating $O\left(\epsilon^{2}\right)$ terms we obtain two analogous systems of equations for $a_{m}$ and $b_{m}$, the former expressible in the form:

$$
\left(\hat{\mathcal{L}}_{A}-\partial_{t}\right) \vec{a}=-\left(\partial_{\tau} x_{A}\right) \vec{\xi}_{A}+\overrightarrow{\mathcal{I}} / \epsilon
$$

Here $\vec{a}=\left(R e a_{1}, R e a_{2}, I m a_{1}, I m a_{2}\right)^{T}$; operator $\hat{\mathcal{L}}_{A}$ is the linearization of Eqs. (1) around the soliton $A_{m} ; \vec{\xi}_{A}=$ $\partial_{x}\left(\operatorname{Re} A_{1}, \operatorname{Re} A_{2}, \operatorname{Im} A_{1}, \operatorname{Im} A_{2}\right)^{T}$ is the neutral eigenmode of $\hat{\mathcal{L}}_{A}$ associated with translational symmetry, $\hat{\mathcal{L}}_{A} \vec{\xi}_{A}=0$; and $\overrightarrow{\mathcal{I}}=\left(-\operatorname{Im} \mathcal{I}_{1},-\operatorname{Im} \mathcal{I}_{2}, \operatorname{Re} \mathcal{I}_{1}, \operatorname{Re} \mathcal{I}_{2}\right)^{T}$ controls the interaction of the two CS.

The solution of Eq. (3) should in general be expressed as a superposition of the eigenmodes $\vec{\xi}_{n}$ of $\hat{\mathcal{L}}_{A}, \hat{\mathcal{L}}_{A} \vec{\xi}_{n}=$ $\lambda_{n} \vec{\xi}_{n}$, with time dependent coefficients, because the CS interaction will couple to them all. However, apart from the above-mentioned neutral eigenmodes, the only analytic knowledge about the eigensystems of $\hat{\mathcal{L}}_{A}$ and $\hat{\mathcal{L}}_{B}$ is that they have two bands of continuum modes with eigenvalues $\lambda$ lying on $\operatorname{Re} \lambda=-\gamma_{m}$, i.e. that all extended eigenmodes are damped. We have obtained their full eigensystems numerically, using finite-differences, over wide ranges of all relevant parameters. We find that for sufficiently large dissipation all cavity solitons are stable throughout the entire region of their existence. A Hopf bifurcation can occur as photon lifetime is increased, but we will not consider here any parameter regions where isolated CS are unstable. With oscillatory eigenmodes absent or well damped, only the neutral mode is easily excited by external perturbations, and so we meantime neglect all other modes. This enables us to obtain semianalytic results on CS interactions. 
To exclude secularly growing solutions the right-hand side of Eq. (3) must be orthogonal to the neutral eigenmode of $\hat{\mathcal{L}}_{A}^{\dagger}$ (which we calculated numerically). This solvability condition, together with that for the $B$ soliton, defines a function $f$ which governs the dynamical evolution of the distance $d$ between the soliton centers:

$$
\partial_{t} d=f(d)
$$

We computed $f$ for both in-phase and out-of-phase interacting CS, for many parameter values. Typical examples are plotted in Fig. 1. Regions where $f$ is negative (positive) correspond to CS attraction (repulsion). Zeros of $f(d)$ thus mark stationary bound states of CS pairs, which are stable if $\partial_{d} f<0$ where $f=0$.

We find that this equation gives generally correct predictions of the inter-soliton forces, in particular that inphase CS attract and out-of-phase CS repel. Both repulsion and attraction become stronger as $\mu$ increases, presumably because the signal component $\left(E_{1}\right)$ of the CS becomes less localized as $\mu$ approaches plane-wave threshold at $\mu_{R}$. A similar effect can be envisaged in other CS models. For in-phase CS the function $f$ can develop pairs of zeros, see Fig. 1. This predicts birth of new pairs of CS bound states, one stable and one unstable.

In Fig. 2 we present simulation results showing different interaction scenarios for two CS initially separated by about three soliton widths. First, we consider interaction of in-phase solitons. For small $\mu$ mutual attraction results in fusion of two solitons into one (Fig. 2(a)). Gradually increasing $\mu$ we first observe formation of a stable oscillatory bound state (Fig. 2(b)), then of a stationary bound state (Fig. 2(c)) which is stable (the radiation visible in Fig. 2(c) decays, albeit slowly). Note that the equilibrium separation in Fig. 2(c) is predicted quite well by the appropriate zero of $f(d)$ in Fig. 1, even though these CS are close enough to endanger the assumptions of our perturbation method. Stationary two-hump solitary states have been found previously [3] as solutions of an approximate equation derived from Eqs. (1), but no analysis of soliton interactions was performed. Note that we have found not only two-hump but also multi-hump solitary states. However the latter were usually dynamically unstable. Further details on this issue will be reported elsewhere. Close to the upper boundary of CS existence the interaction of two solitons excites a global pattern, see Fig. 2(d), via generation of a switching wave from the stable trivial solution up into the modulationally unstable nontrivial homogeneous state. As predicted by Eq. (4), out-of-phase CS repel each other throughout the entire region of their existence - contrast Fig. 2(e) with Fig. 2(d), which corresponds to the same value of $\mu$.

Now we will describe numerical results of the interaction of CS where weakly-damped oscillatory modes strongly influence the soliton interactions. Oscillating solitons generally radiate energy, which can become trapped between neighbouring solitons, exerting a radia- tion force which may lead to formation of a bound state. An effect of this kind has been reported for solitons in models with a weak global dissipation [7]. We investigated a quite different situation, where linear waves escaping from the soliton are strongly damped. Here strong interaction between the solitons is due, not to radiation modes, but to proto-Hopf modes, and thus has novel aspects.

The effect is strong providing that two conditions are satisfied. First, and crucially, the corresponding eigenmodes must have tails with well pronounced and weakly decaying oscillatory structure, see Fig. 3(a). Second, as might be expected, the oscillatory mode should be weakly damped (see Fig. 3(b)), i.e. the CS is close to a Hopf instability. If both conditions hold, then, even if the global damping due to the $\gamma_{m}$ is strong, a CS acts as a guide for waves weakly damped in both space and time. If a second CS is close enough, these guided waves can couple and reinforce each other. Fig. 3(c, d) illustrates the dynamics of two interacting CS having eigenmodes shown in Fig. 3(a). Note that the separation of the interacting solitons in Fig. 3(c) is much greater than their width. Comparison with Fig. 3(b) clearly indicates that the undamped pulsations shown in Fig. 3(d) originate from coupling and mutual reinforcement of the oscillatory modes of the two solitons. A further interesting point is that we find these dynamic bound states also for out-of-phase solitons, balancing the repulsion induced by their neutral mode interaction.

Quadratic nonlinearity is also known to support solitons in free propagation geometry and in particularly interaction of these solitons has recently been studied both experimentally and theoretically 8,9.9. Hamiltonian nature of free propagating solitons results in their interaction obeying the laws of Newtonian dynamics [9]. Another important difference is that the relative phase of the interacting solitons can take only two discrete values in a cavity while it is a continuous free parameter in a propagation scheme. In spite of these differences fusion of the in-phase solitons and repulsion of the out-phase are common features in both schemes. However, existence of the stationary and oscillatory bound states coupled either via translational neutral modes or via internal oscillatory modes are novel important features arising due to presence of the external pump and cavity losses. Balance between the pump and losses acts as an additional, equally important with the balance between diffraction and nonlinearity, mechanism of soliton formation inside an optical cavity.

In summary, we have presented the analytical and numerical study of the interaction of cavity solitons in a degenerate $\mathrm{OPO}$ and identified distinct static and dynamic binding mechanisms.

D.V.S. thanks C. Etrich, F. Lederer, D. Michaelis and U. Peschel for warm hospitality and illuminating discussions of many relevant questions during his short visit to Jena. He also acknowledges financial support from the Royal Society of Edinburgh and British Petroleum. The 
work is partially supported by ESPRIT project PIANOS and EPSRC grant GR/M19727.

[1] N.N. Rosanov, Progress in Optics 35, 1 (1996); M. Tlidi, P. Mandel, and R. Lefever, Phys. Rev. Lett. 73, 640 (1994); W.J. Firth and A.J. Scroggie, Phys. Rev. Lett. 76, 1623. For a recent review of CS, see W.J. Firth and G.K. Harkness, Asian J. Phys. 7, 665 (1998).

[2] M. Brambilla, L.A. Lugiato, F. Prati, L. Spinelli, and W.J. Firth, Phys. Rev. Lett. 79, 2042 (1997); D. Michaelis, U. Peschel, and F. Lederer, Phys. Rev. A 56, R3366 (1997).

[3] K. Staliunas and V.J. Sánchez-Morcillo, Opt. Commun. 139, 306 (1997); S. Longhi, Phys. Scripta 56, 611 (1997); S. Trillo and M. Haelterman, Opt. Lett. 23, 1514 (1998).

[4] C. Etrich, U. Peschel, and F. Lederer, Phys. Rev. Lett. 79, 2454 (1997); S. Longhi, Opt. Lett. 23, 346 (1998).

[5] C. Richy, K.I. Petsas, E. Giacobino, C. Fabre, and L. Lugiato, J. Opt. Soc. Am. B 12, 456 (1995); A.G. White, J. Mlynek, and S. Schiller, Europhys. Lett. 35, 425 (1996).

[6] Various versions of this method can be found, e.g., in: K. A. Gorshkov and L. A. Ostrovsky, Physica D 3, 428 (1981); S. Longhi, Phys. Rev. E 55, 1060 (1997).

[7] B.A. Malomed, Phys. Rev. E 47, 2874 (1993).

[8] C. Etrich, U. Peschel, F. Lederer, and B. Malomed, Phys. Rev. A 52, R3444 (1995); C.B. Clausen, P.L. Christiansen, and L. Torner, Opt. Commun. 136, 185 (1997); Y. Baek, R. Schiek, G.I. Stegeman, I. Baumann, and W. Sohler, Opt. Lett. 22, 1550 (1997); D.M. Baboiu, G.I. Stegeman, Opt. Quant. Electr. 30, 849 (1998); A. Barthelemy, B. Bourliaguet, V. Kermene, B. Costantini, C. De Angelis, D. Modotto, and G. Assanto, Opt. Quant. Electr. 30, 923 (1998).

[9] V.V. Steblina, Y.S. Kivshar, and A.V. Buryak, Opt. Lett. 23, 156 (1998); A.V. Buryak and V.V. Steblina, J. Opt. Soc. Am. B 16, 245 (1999).

[10] Only the central third of the computational window in $x$ is shown in Figs. 2(a)-(c),(e), 3(c).

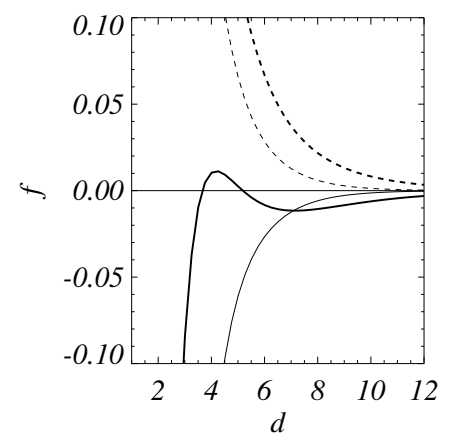

FIG. 1. Plots of the CS velocity function $f$ vs $d$. Full (dashed) lines correspond to in-phase (out-of-phase) solitons and thin (thick) lines correspond to $\mu=1.6(1.9)$. Other parameters are $\delta_{2}=-4, \delta_{1}=-1.8, \gamma_{1}=1, \gamma_{2}=0.8$.

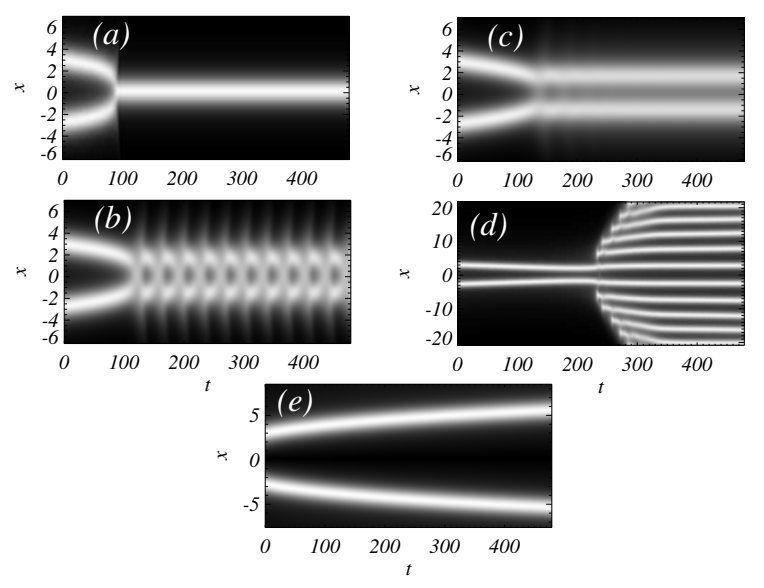

FIG. 2. Interaction dynamics of $\chi^{(2)}$ cavity solitons [9]. At different values of pump parameter $\mu$, in-phase CS: (a) merge, $\mu=1.6$; (b) form oscillatory bound state, $\mu=1.8$; (c) form stable stationary bound state, $\mu=1.9$; (d) generate a pattern via a switching wave, $\mu=2$. Out-of-phase solitons repel, e.g. at $\mu=2$, (e). Other parameters as for Fig. 1.
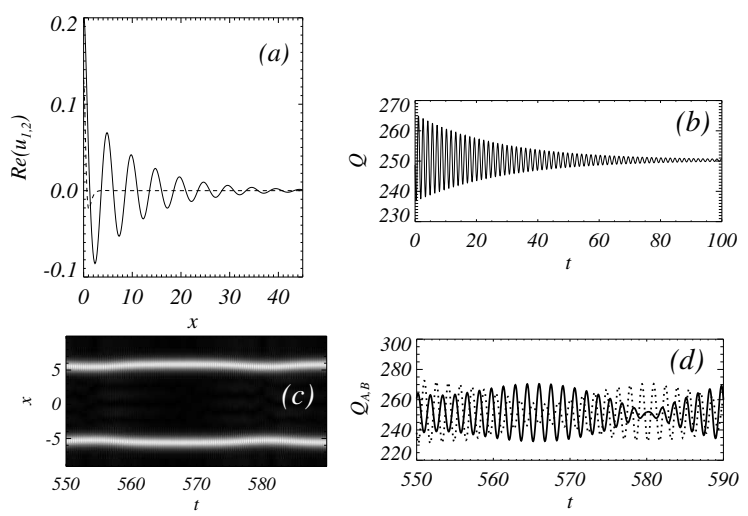

FIG. 3. Dynamic interaction of CS for $\mu=2, \delta_{1}=-3$, $\delta_{2}=-12, \gamma_{1}=0.3, \gamma_{2}=1$, for which the CS has a mode with eigenvalue pair $\lambda \simeq-0.03 \pm i 4.14$ : (a) Spatial structure of the eigenmode, $\operatorname{Re}\left(u_{1}\right)$ - full lines, $\operatorname{Re}\left(u_{2}\right)$-dashed lines; (b) Temporal evolution of signal energy $Q=\int d x\left|E_{1}\right|^{2}$ for slightly perturbed single soliton, showing damped oscillation; (c) Spatio-temporal evolution [9] of $\left|E_{1}\right|$ (time window much later than in (b)), showing dynamic bound state; (d) Temporal evolution of signal energies of the two CS in (c), in the same time window, showing rapid undamped oscillations and slow energy exchange between the two solitons. 